\title{
Diagnosis of occult scaphoid fractures: a randomized, controlled trial comparing bone scans to radiographs for diagnosis
}

\author{
Christina Hiscox, MD, FRCSC*; Jeremy LaMothe, MD, PhD*; Neil White, MD*; Mark Bromley, MD; \\ Elizabeth Oddone Paolucci, BA, MSc, PhD; Kevin Hildebrand, MD*
}

\section{ABSTRACT}

Background: Many patients with suspected scaphoid fractures but negative radiographs are immobilized for $\geq 2$ weeks and are eventually found to have no fracture. Bone scans are reportedly $99 \%$ sensitive for these injuries if done $\geq 72$ hours postinjury.

Objective: The purpose of this study was to determine if early bone scans would allow for shorter cast immobilization periods in patients with suspected scaphoid fractures.

Methods: Twenty-seven patients with clinically suspected scaphoid fractures and negative radiographs were randomized to early diagnosis (bone scan within 3-5 days; $n=12$ ) or traditional diagnosis (radiographs 10-14 days postinjury; $n=$ 15). The primary outcome was number of days immobilized in a cast.

Results: The mean number of days immobilized was 26 in the traditional group and 29 in the bone scan group. Overall, 6 patients had scaphoid fractures (2 in the traditional diagnosis group and 4 in the bone scan group; $p>0.05$ ), and 8 had other types of fractures. These other types of fractures included four distal radius fractures, two triquetral fractures, one trapezoid fracture, and one hamate fracture. There was no significant difference in the number of other types of fractures between groups. The Kaplan-Meier survival analysis using the log-rank test revealed that there was no statistically significant difference between days immobilized between the radiograph and bone scan groups $(p=0.38)$.

Conclusions: The current study suggests that the use of bone scans to help diagnose occult scaphoid fractures does not reduce the number of days immobilized and that the differential diagnosis of occult scaphoid fractures should remain broad because other injuries are common.

\section{RÉSUMÉ}

Contexte: Bien souvent, il y a immobilisation du poignet pendant $\geq 2$ semaines, pour des fractures présumées du scaphoïde, malgré des radiographies négatives, et I'on constate finalement l'absence de fracture. La scintigraphie osseuse aurait une sensibilité de $99 \%$ à l'égard de ces lésions si elle est effectuée $\geq 72$ heures après l'accident.

Objectif: L'étude visait à déterminer si le recours à une scintigraphie osseuse précoce permettrait d'écourter la période d'immobilisation plâtrée chez les patients souffrant d'une fracture présumée du scaphoïde.

Méthode: Vingt-sept patients qui souffraient d'une fracture présumée du scaphoïde d'après les signes cliniques et chez qui les radiographies étaient négatives ont été dirigés au hasard vers le groupe de diagnostic précoce (scintigraphie osseuse: 3-5 jours; $n=12$ ) ou vers le groupe de diagnostic usuel (radiographie: $10-14$ jours; $n=15$ ). Le principal critère d'évaluation était le nombre de jours d'immobilisation plâtrée.

Résultats: Le nombre moyen de jours d'immobilisation du poignet était de 26 dans le groupe de diagnostic usuel et de 29 dans le groupe de diagnostic précoce. Dans l'ensemble, 6 patients souffraient d'une fracture du scaphoïde (2 dans le groupe de diagnostic usuel; 4 dans le groupe de diagnostic précoce; $p>0.05$ ) et 8 , d'autres types de fracture. Ces derniers comprenaient quatre fractures du radius distal, deux fractures du pyramidal, une fracture du trapézoïde et une fracture de l'os crochu. II n'y avait pas d'écart significatif quant au nombre d'autres types de fractures entre les deux groupes. L'analyse de survie selon la méthode de KaplanMeier, réalisée à l'aide du test logarithmique par rangs a révélé l'absence d'écart statistiquement significatif du nombre de jours d'immobilisation entre le groupe de radiographie et celui de la scintigraphie osseuse $(p=0.38)$.

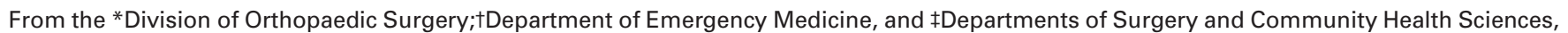
University of Calgary, Calgary, AB.

Correspondence to: Dr. C. Hiscox, Division of Orthopaedic Surgery, University of Calgary, Health Sciences Centre, 3280 Hospital Drive NW, Calgary, AB T2N 4Z6; chiscox71@hotmail.com.

This article has been peer reviewed. 
Conclusions: D'après les résultats de l'étude, le recours à la scintigraphie osseuse aux fins de diagnostic de fractures occultes du scaphoïde ne permet pas de diminuer le nombre de jours d'immobilisation, et l'éventail de diagnostics différentiels de la fracture du scaphoïde devrait rester large en raison de la fréquence élevée d'autres types de lésions.

Keywords: diagnosis, fracture, scaphoid
With carpal injuries, the scaphoid is the bone most often fractured. ${ }^{1}$ Complications with scaphoid fractures arise because of the scaphoid's vulnerable blood supply, irregular shape, oblique alignment across the plane of motion of the midcarpal joint, and complex function as a connection between the proximal and distal carpal rows. ${ }^{2}$ Nonunion of the scaphoid can result in major changes in the kinematics of the wrist, ultimately resulting in disabling wrist pain. ${ }^{3,4}$ Early treatment of scaphoid fractures with immobilization is critical to gain union and avoid late complications. However, diagnosing scaphoid fractures may be difficult, especially when the clinical examination suggests a scaphoid fracture (tender "snuff box"), but the radiographs do not reveal a fracture. A normal radiograph in a patient with snuffbox tenderness is known as a clinical scaphoid fracture. In this setting, if the patient does have a scaphoid fracture, the injury is termed an occult scaphoid fracture. Approximately 80 to $97 \%$ of patients with a clinical scaphoid fracture do not have a scaphoid fracture. ${ }^{2,5-11}$

The traditional approach to the patient with a clinical scaphoid fracture consists of immediate application of a thumb spica cast or splint and re-evaluation of the wrist after a 10- to 14-day period of immobilization. If tenderness persists, radiographs are repeated to assess for a scaphoid fracture. Unfortunately, repeat radiographs are only 30\% sensitive, ${ }^{12-15}$ so the patient again returns for repeat radiographs after another 10 to 14 days or undergoes a three-phase bone scan. Radiographs or bone scans suggesting a scaphoid fracture result in a further 4 to 10 weeks of immobilization. Thus, patients are immobilized for at least 2 weeks based on a physical examination finding (snuff-box tenderness) of limited specificity. This approach immobilizes a large number of patients needlessly.

Bone scans around 3 days after injury offer the possibility of early diagnosis and may allow earlier wrist mobilization for those who do not have a fracture. Bone scans after 3 days are as sensitive and specific as bone scans after 14 days $^{2,5,16}$ and more sensitive than plain radiographs.

The purpose of this study was to determine if an early bone scan would decrease the number of days that patients were immobilized with clinical scaphoid fractures. We hypothesized that clinical scaphoid fracture patients who received early bone scanning would be immobilized for a shorter duration when compared to patients who received the routine practice of serial clinical examinations and radiography.

\section{METHODS}

\section{Study design and setting}

This was an open-label, randomized, controlled clinical trial. Patients with clinical scaphoid fractures who presented to two emergency departments (EDs) in Calgary, Alberta (Foothills Medical Centre and the Peter Lougheed Centre), between September 2008 and April 2009 were invited to participate in the study. The two hospitals participating in the study are teaching hospitals with $>600$-bed capacity each, with a combined annual ED census of 135,000 patients per year. The diagnosis of a clinical scaphoid fracture was made by the attending emergency physician based on an acute wrist injury with snuff-box tenderness and normal radiographs. The emergency physician contacted the study team to assess potentially eligible subjects for enrolment into the study.

\section{Study population}

All patients between 18 and 80 years old presenting to the ED within 48 hours of their injury and referred by the emergency physician for a clinical scaphoid fracture were assessed by the study team. Patients with ipsilateral upper extremity fractures diagnosed at the time of presentation and women who were breastfeeding or pregnant were excluded.

\section{Enrolment}

Patients eligible for the study were consented and enrolled by the study team. The study team was composed of an emergency medicine resident, orthopedic surgery residents, and a medical student. A member of the study team was available for the 
emergency physician to contact via a research pager 24 hours per day, 7 days per week.

\section{Randomization}

Immediately after patients were consented, the patients were randomized using an online randomization service $(<$ http:www.sealedenvelope.com/ $>$ ) to either the traditional radiograph group or the early bone scan group in a 1:1 ratio in random permuted blocks. There was no stratification by site.

\section{Outcomes}

After being randomized to their respective treatment group, patients from both groups were placed in wellmoulded, below-elbow thumb spica casts and discharged from hospital. Patients in the radiograph group followed up with the ED 10 to 14 days after the injury for repeat physical examination and radiography. If they were diagnosed with a fracture, they were treated specifically for the fracture with further immobilization. Patients with negative radiographs and snuff-box tenderness received either bone scanning or further immobilization with follow-up in 10 to 14 days at the discretion of the treating emergency physician. Patients in the early bone scan group had bone scanning done 3 to 5 days after the injury. On the day of the bone scanning, patients went to the ED for the results of the bone scan from the emergency physician. If the bone scan was positive, the injured wrist continued to be immobilized. If the bone scan was negative, the wrist was no longer immobilized (Figure 1).

All patients in the study were requested to follow up with one orthopedic surgeon in the cast clinic at 6 weeks and 12 months after their injury. Patients diagnosed with a fracture were seen more frequently. Repeat radiography and physical examination were done at the cast clinic visits. This was done by a physician blind to the study group. Data collected included the number of days immobilized, type of injury diagnosed, range of motion measured with a goniometer, and grip strength using a dynamometer. The primary outcome measured was days of wrist immobilization.

\section{Analysis}

$G^{*}$ Power 3.1.3 software (Institute for Experimental Psychology, Düsseldorf, Germany) was used to perform a power and sample size calculation. The study was powered to display a difference in days immobilized of 5. This translates to a 5-day work week and

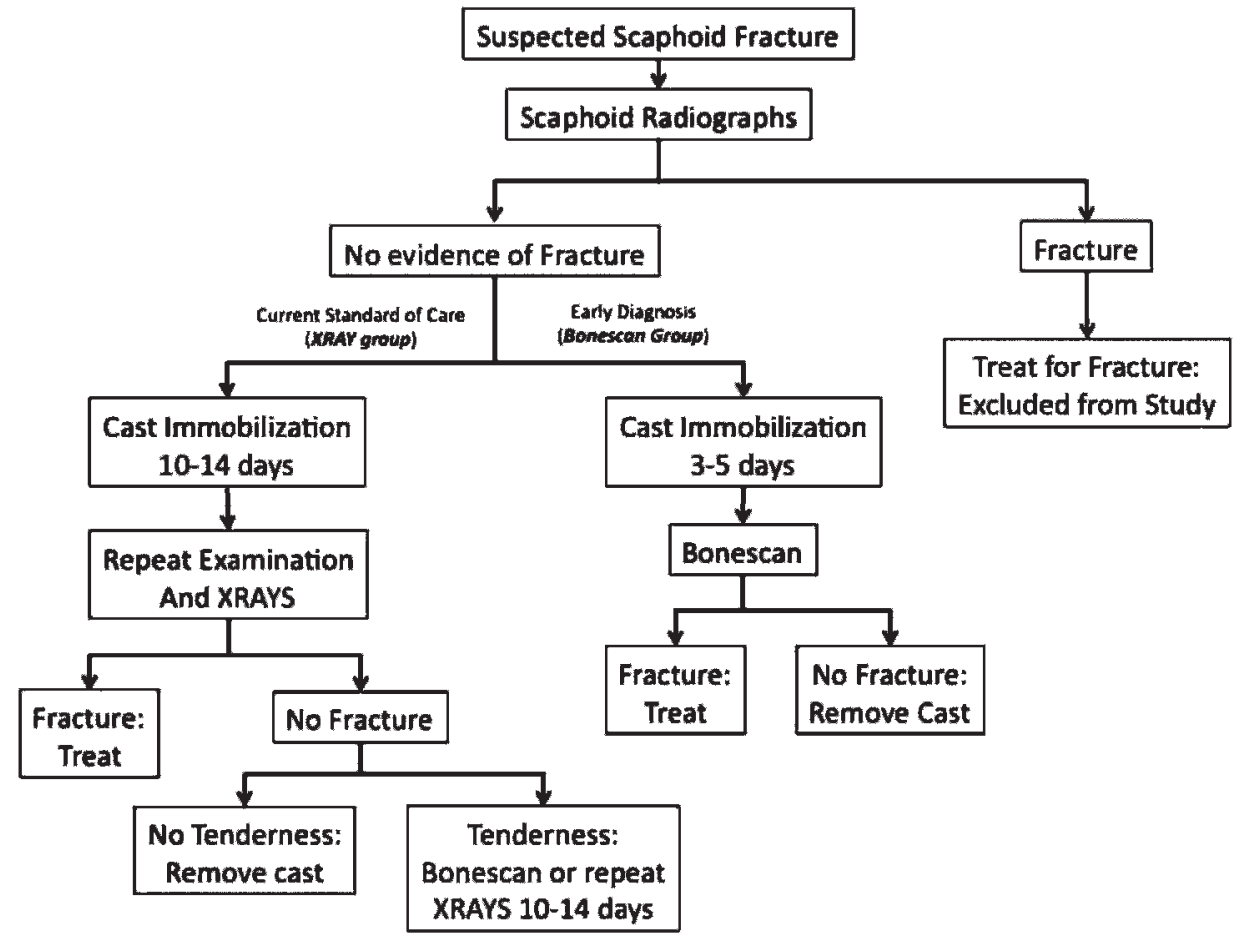

Figure 1. Clinical treatment pathway. 
was believed to be clinically meaningful. Between 1 and $20 \%$ of clinical scaphoid fractures have an occult fracture $^{12-15,17}$; thus, the sample size calculation was based on $10 \%$ scaphoid fractures in each group. We assumed that patients with a suspected scaphoid fracture did not have any other type of fracture. Based on the aforementioned treatment protocol, it was estimated that the variability within each group was 4 and the average number of days immobilized would be 5 days for patients in the bone scan group and 12 days in the radiograph group for patients without a fracture. Thus, a total sample size of 10 patients $\left(n_{1}=5, n_{2}=5\right)$ would be required when testing for a one-tailed difference between two independent group means, using an alpha of 0.05 , power of 0.8 , and effect size $(d)$ of 1.75 . All protocols were approved by our institutional ethics review board.

All statistical analyses were performed using $I B M$ SPSS Statistics for Windows, version 19.0 (IBM Corp., Armonk, NY), and included the calculation of descriptive statistics, cross-tabulation $\chi^{2}$ analyses, independent measures $t$-tests, and Kaplan-Meier survival analysis.

\section{RESULTS}

Thirty patients were assessed for study inclusion between September 2008 and April 2009. Twentyseven patients were enrolled in the study (Figure 2). Two patients declined, and one was excluded (too young). Fifteen patients were enrolled in the radiograph group and 12 in the bone scan group. There were 16 males and 11 females. The mean age was 36 years. No statistical differences were found between groups in terms of age, sex, or dominant hand involved (Table 1). One patient allocated to the radiograph group had a bone scan. He was found to have a trapezoid fracture and was analyzed as part of the radiograph group on the basis of the intention to treat. All patients in the study were available for measurement of the primary outcome (number of days immobilized) and were deemed to have a final diagnosis of fracture or no fracture based on a final examination at the time their casts were removed.

The median (interquartile range) number of days immobilized was $15(5,55)$ days in the bone scan group and $13(10,42)$ days in the radiograph group (overall
ENROLLMENT

ALLOCATION

\section{Assessed for Eligibility ( $n=30$ )}

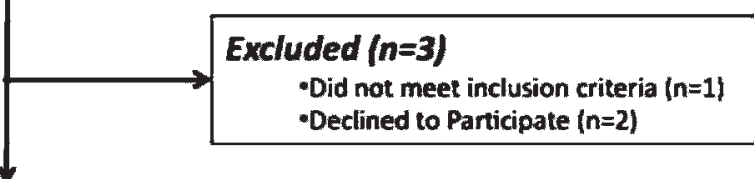

- Declined to Participate ( $n=2)$

Randomized ( $n=27)$

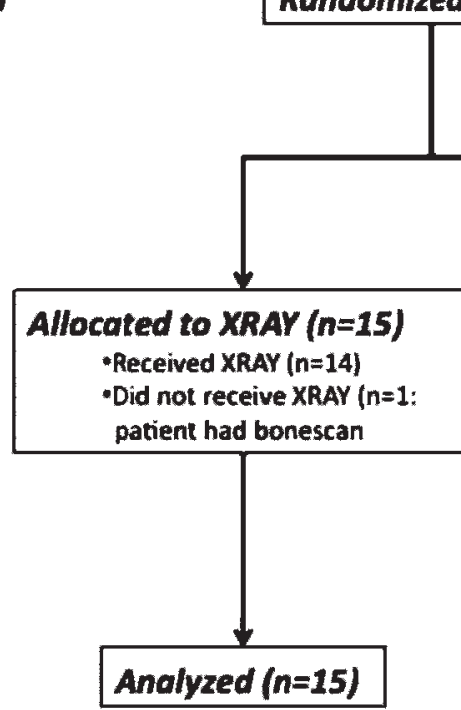

ANALYSIS

Analyzed ( $n=15)$

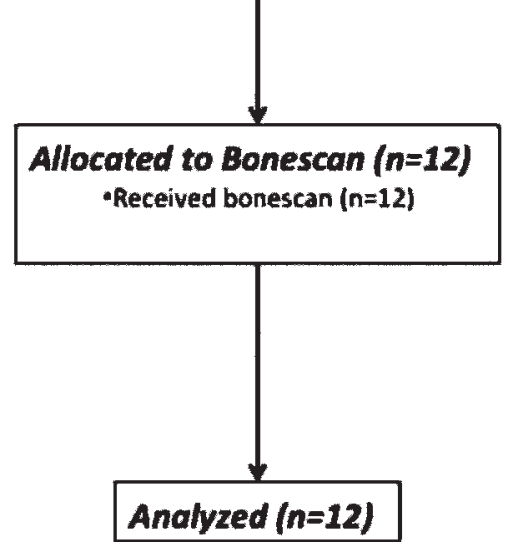

Analyzed ( $n=12)$

Figure 2. Patient treatment flow diagram. 


\begin{tabular}{|lccc|}
\hline Table 1. Patient demographics & & \\
\hline & Overall $(n=27)$ & Radiograph $(n=15)$ & Bone scan $(n=12)$ \\
\hline Age, $y r$ & & & $35.9(12.4)$ \\
Mean (SD) & $35.9(12.8)$ & 25 & 22.3 \\
25th percentile & 25 & 33 & 34.5 \\
50th percentile & 33 & 44 & 49 \\
75th percentile & 49 & 20 & 18 \\
Minimum & 18 & 61 & 58 \\
Maximum & 61 & $8 / 7$ & $8 / 4$ \\
Male/female & $16 / 11$ & 8 & 6 \\
Dominant hand & 14 & & \\
\hline
\end{tabular}

range of 4-84 days; Table 2). There was no difference between groups in the length of immobilization $(p=$ 0.38 by log-rank survival analysis). Figure 3 illustrates the Kaplan-Meier survival curve for time until cast removed by group.

With regards to the identification of any fracture, 7 of $12(58 \%)$ of the bone scan group and 7 of 15 (47\%) of the radiograph group were ultimately diagnosed with a fracture ( $p=0.55$ by Pearson $\chi^{2}$ analysis). Six of the 27 patients in the study had a scaphoid fracture. Four of these patients were in the bone scan group and two were in the radiograph group (Table 3). The remaining fractures consisted of two triquetral and one hamate fracture in the bone scan group and four distal radius and one trapezoid fracture in the radiograph group. None of these fractures occurred concurrently with a scaphoid fracture. The estimated odds ratio for any fracture in the bone scan versus the radiograph group was 1.6 (95\% CI $0.35-7.4)$.

Five of 12 (42\%) patients who had a bone scan did not have a fracture. Three were female (range 18-58 years old), and they were immobilized on average for 6 days (SD = 3). Eight of $15(53 \%)$ patients in the radiograph group did not have a fracture. Four were female (range 26-61 years old), and they were immobilized on average for 12 days $(\mathrm{SD}=4)$.

None of the patients in the radiograph group who had a diagnosis of "no fracture" went on to eventual bone scanning to establish the final diagnosis. The diagnosis of no fracture was made after two sets of radiographs combined with a repeat physical examination.

The long-term follow-up was very limited. Only 3 of the 27 patients followed up at 1 year. Many of the patients who did not follow up were unable to be contacted for future follow-up after repeated attempts from the study team. Eleven patients followed up 6 weeks after their injury. Five of these patients were in the bone scan group and 6 were in the radiograph group. Strength, range of motion, and questionnaire data were not analyzed due to the insufficient follow-up.

\section{DISCUSSION}

The fact that there was no difference in days immobilized in a cast between the two groups is somewhat counterintuitive but may be attributed to the substantial other types of fractures identified in our

Table 2. Days immobilized

\begin{tabular}{|c|c|c|c|c|}
\hline Days immobilized & Overall $(n=27)$ & Radiograph $(n=15)$ & Bone scan $(n=12)$ & $p$ \\
\hline Mean (SD) & $27(24)$ & $26(20)$ & $29(29)$ & 0.35 \\
\hline 25th percentile & 10 & 10 & 5 & \\
\hline 50th percentile & 13 & 13 & 15 & \\
\hline 75th percentile & 44 & 42 & 55 & \\
\hline Minimum & 4 & 8 & 4 & \\
\hline Maximum & 84 & 77 & 84 & \\
\hline
\end{tabular}






Figure 3. Numbers of days immobilized for radiograph versus bone scan patients.

subjects and the slightly higher number of scaphoid fractures found in the bone scan group. The study was powered on the assumption that only $10 \%$ of patients would have a scaphoid fracture and the rest are immobilized unnecessarily. However, in the current study, half of the subjects had fractures and received at least 6 weeks' immobilization.

That many patients had fractures other than scaphoid fractures makes the early diagnosis of occult scaphoid fractures less valuable because more patients required immobilization for their injury. That other fractures are relatively common with the provisional diagnosis of a suspected scaphoid fracture supports the current literature. Beeres and colleagues examined 50 patients with signs on physical examination of a scaphoid facture and negative radiographs. ${ }^{18}$ Bone scans in their study identified 32\% scaphoid fractures and $40 \%$ other types of occult fractures. Gäbler and colleagues studied 121 patients who presented to the ED with clinical symptoms of an occult scaphoid fracture and negative radiographs. ${ }^{19}$ These patients were followed with repeat radiography and magnetic resonance imaging (MRI). In this study, 83 fractures in 82 patients were identified, and 28 were scaphoid fractures. The remainder were carpal, metacarpal, and distal radius fractures. Seven of these patients sustained two or more bone injuries. Thirty-nine patients had no bone or soft tissue injury identified. Fowler and colleagues compared bone scanning to MRI for diagnosing occult scaphoid fractures..$^{20}$ Of 43 patients,

\begin{tabular}{|lccc|}
\hline Table 3. Fracture by group & & \\
\hline & Overall & Radiograph, $n(\%)(n=15)$ & Bone scan, $n(\%)(n=12)$ \\
\hline No fracture & 13 & $8(53)$ & $5(42)$ \\
Scaphoid fracture & 6 & $2(13)$ & $4(33)$ \\
Other type of fracture & 8 & $5(27)$ & $3(25)$ \\
Total fractures & 14 & $7(47)$ & $7(58)$
\end{tabular}


they identified 6 patients with scaphoid fractures and 6 with other types of fractures. Beeres and colleagues evaluated bone scans to identify occult scaphoid fractures. ${ }^{21}$ In 56 patients, they identified 15 scaphoid fractures and 23 other types of fractures, including distal radius $(n=11)$, other carpal bones $(n=9)$, and metacarpal bones $(n=3)$. In total, 38 of 56 patients had a fracture.

It is interesting to note that many physicians seem to have a high clinical suspicion for occult scaphoid fractures but do not seem to have the same index of suspicion for other occult fractures in the wrist area. This may be due to the poor outcome of undiagnosed scaphoid fractures and relatively better prognosis for other types of wrist fractures. Our study was designed to include the emergency physician's assessment of a clinical scaphoid fracture, thereby representing the real-world environment of injury diagnosis. Accordingly, bone scanning was chosen rather than MRI or computed tomography (CT). MRI is difficult to obtain for all patients with a suspected scaphoid fracture on an urgent basis in the Canadian health care system. Bone scanning was chosen over CT due to its higher sensitivity. ${ }^{22}$

This study was unable to show a difference in days immobilized using an early bone scan for diagnosis; however, the current study demonstrated that patients diagnosed with a clinical scaphoid fracture may often have another type of fracture. It is important to have a high index of suspicion for these other types of fractures. It should be noted that the patients who were enrolled in this study and found to have some type of fracture did have specific snuff-box tenderness. The examination was done by experienced emergency physicians in a large urban setting. The high rate of fracture seen in our group should not be generalized to the patient with a nonspecific sore wrist.

\section{LIMITATIONS}

There are several limitations to our study. The two groups did not have the same proportion of patients with scaphoid fractures despite randomization into treatment groups. In this study, the proportion of patients in each group with a fracture did influence the primary outcome, days immobilized in a cast. It is possible that the larger number of scaphoid fractures identified in the bone scan group could have been due to the higher sensitivity of bone scanning to diagnose these fractures. Many of the patients in the radiograph group did not receive further imaging after they were determined not to have a scaphoid fracture. Having all the patients in the radiograph group receive bone scanning may have helped gain certainty that these patients did not have a fracture. We accepted the traditional teaching that no tenderness and negative radiographs at 10 to 14 days rule out a fracture. We are unaware of any missed fractures in our study groups. The power calculation was based on the incidence of occult scaphoid fractures only and did not consider the incidence of other fractures. Our power calculation was also based on the assumption that 5 days difference of cast immobilization between groups is clinically important. An argument could be made that 5 days is not clinically relevant, especially for the person who can work in a cast. However, many people who can work in a cast may still have trouble with transportation to work as they cannot drive in a cast.

A 12-month follow-up was scheduled for all patients in the study to ensure that there were no missed injuries or nonunions. However, most patients were lost to 12-month follow-up (only three patients returned). This precluded further functional and clinical outcome measurements. This may be because patients without fractures or with asymptomatic healed fractures did not want to return to a follow-up clinic. Many patients were also unreachable for booking a follow-up appointment despite multiple attempts from the study team.

\section{CONCLUSIONS}

The early use of bone scans to confirm the diagnosis of a clinically suspected scaphoid fracture did not significantly reduce the duration of immobilization. More than half of the patients undergoing bone scans had scaphoid or other fractures requiring immobilization.

Competing interests: This study was supported by two grants from the Canadian Orthopaedic Foundation, the Hip Hip Hooray Grant, and the Community Investment Fund Research Grant.

\section{REFERENCES}

1. Kozin SH. Incidence, mechanism, and natural history of scaphoid fractures. Hand Clin 2001;17:515-24.

2. Chakravarty D, Sloan J, Brenchley J. Risk reduction through skeletal scintigraphy as a screening tool in suspected 
scaphoid fracture: a literature review. Emerg Med 7 2002;19: 507-9, doi:10.1136/emj.19.6.507.

3. Leventhal EL, Wolfe SW, Moore DC, et al. Interfragmentary motion in patients with scaphoid nonunion. F Hand Surg Am 2008;33:1108-15, doi:10.1016/ j.jhsa.2008.03.008.

4. Moritomo H, Murase T, Oka K, et al. Relationship between the fracture location and the kinematic pattern in scaphoid nonunion. 7 Hand Surg Am 2008;33:1459-68, doi:10.1016/ i.jhsa.2008.05.035.

5. Murphy D, Eisenhauer M. The utility of a bone scan in the diagnosis of clinical scaphoid fracture. 7 Emerg Med 1994;12: 709-12, doi:10.1016/0736-4679(94)90432-4.

6. Murphy DG, Eisenhauer MA, Powe J, et al. Can a day 4 bone scan accurately determine the presence or absence of scaphoid fracture? Ann Emerg Med 1995;26:434-8, doi:10. 1016/S0196-0644(95)70110-9.

7. Mittal RL, Dargan SK. Occult scaphoid fracture: a diagnostic enigma. 7 Orthop Trauma 1989;3:306-8, doi:10. 1097/00005131-198912000-00008.

8. Waeckerle JF. A prospective study identifying the sensitivity of radiographic findings and the efficacy of clinical findings in carpal navicular fractures. Ann Emerg Med 1987;16:733-7, doi:10.1016/S0196-0644(87)80563-2.

9. Waizenegger M, Wastie ML, Barton NJ, et al. Scintigraphy in the evaluation of the "clinical" scaphoid fracture. $\mathcal{F}$ Hand Surg Br 1994;19:750-3, doi:10.1016/0266-7681(94)90251-8.

10. Schubert HE. Scaphoid fracture. Review of diagnostic tests and treatment. Can Fam Physician 2000;46:1825-32.

11. Roolker W, Maas M, Broekhuizen AH. Diagnosis and treatment of scaphoid fractures, can non-union be prevented? Arch Orthop Trauma Surg 1999;119:428-31, doi:10. 1007/s004020050014.

12. Rolfe EB, Garvie NW, Khan MA, et al. Isotope bone imaging in suspected scaphoid trauma. Br 7 Radiol 1981;54: 762-7, doi:10.1259/0007-1285-54-645-762.
13. Zarnett R, Martin C, Barrington TW, et al. The natural history of suspected scaphoid fractures. Can 7 Surg 1991;34:334-7.

14. Leslie IJ, Dickson RA. The fractured carpal scaphoid. Natural history and factors influencing outcome. $\mathcal{f}$ Bone Foint Surg Br 1981;63-B:225-30.

15. Duncan DS, Thurston AJ. Clinical fracture of the carpal scaphoid—an illusionary diagnosis. 7 Hand Surg $\mathrm{Br}$ 1985;10: 375-6, doi:10.1016/S0266-7681(85)80065-6.

16. Tiel-van Buul MM, Broekhuizen TH, van Beek EJ, et al. Choosing a strategy for the diagnostic management of suspected scaphoid fracture: a cost-effectiveness analysis. 7 Nucl Med 1995;36:45-8.

17. Tiel-van Buul MM, Roolker W, Broekhuizen AH, et al. The diagnostic management of suspected scaphoid fracture. Injury 1997;28:1-8, doi:10.1016/S0020-1383(96)00127-1.

18. Beeres FJP, Hogervorst M, Rhemrev SJ, et al. A prospective comparison for suspected scaphoid fractures: bone scintigraphy versus clinical outcome. Injury 2007;38:769-74, doi:10.1016/j.injury.2006.12.009.

19. Gäbler C, Kukla C, Breitenseher MJ, et al. Diagnosis of occult scaphoid fractures and other wrist injuries. Are repeated clinical examinations and plain radiographs still state of the art? Langenbecks Arch Surg 2001;386:150-4, doi:10.1007/s004230000195.

20. Fowler C, Sullivan B, Williams LA, et al. A comparison of bone scintigraphy and MRI in the early diagnosis of the occult scaphoid waist fracture. Skeletal Radiol 1998;27:683-7, doi: $10.1007 / \mathrm{s} 002560050459$.

21. Beeres FJP, Hogervorst M, den Hollander P, et al. Outcome of routine bone scintigraphy in suspected scaphoid fractures. Injury 2005;36:1233-6, doi:10.1016/j.injury.2005.02.009.

22. Memarsadeghi M, Breitenseher MJ, Schaefer-Prokop C, et al. Occult scaphoid fractures: comparison of multidetector CT and MR imaging - initial experience. Radiology 2006;240: 169-76, doi:10.1148/radiol.2401050412. 